\title{
Ciclo gametogênico e comportamento reprodutivo de Iphigenia brasiliana (Mollusca, Bivalvia, Donacidae) no estuário do rio Subaé, Baía de Todos os Santos, Bahia, Brasil
}

\author{
Patrícia P. Silva ${ }^{1}$; Marlene C. Peso-Aguiar ${ }^{2} \&$ Gabriel Ribeiro $^{3}$
}

1. Plano Nacional de Formação de Professores (PARFOR), Universidade Federal do Recôncavo da Bahia, Rua Rui Barbosa, 710, Centro, 44380-000 Cruz das Almas, BA, Brasil.
(patpetitinga@yahoo.com.br)
2. Departamento de Zoologia, Instituto de Biologia, Universidade Federal da Bahia, Campus Universitário de Ondina s/n, Ondina, 40170-115 Salvador, BA, Brasil. (mpeso@ufba.br)
3. Centro de Ciências Agrárias, Ambientais e Biológicas (CCAAB), Universidade Federal do Recôncavo da Bahia, Campus Universitário, Centro, 44380-000 Cruz das Almas, BA, Brasil.
(fta gabrielribeiro@yahoo.com.br)

\begin{abstract}
Gametogenic cycle and reproductive behavior of Iphigenia brasiliana (Mollusca, Bivalvia, Donacidae) in the Subaé river estuary, Todos os Santos Bay, Bahia, Brazil. This study aimed to describe the gametogenic cycle and reproductive behavior of the population of Iphigenia brasiliana (Lamarck, 1818) in the estuary of the Subaé river, Todos os Santos Bay, Bahia. The bivalves were collected from November 2001 to November 2002. A total of 244 specimens was measured (anteroposterior axis), gutted, fixed, dehydrated and embedded in paraffin. The histology of the gonads was performed by $5 \mathrm{~mm}$ thick serial sections of gonadal tissue, and stained with HE. The length at the beginning of gonadal maturation $(\mathrm{Lpm})$ was estimated from the distribution of the relative frequencies of youth and adults, by length class of individuals. The relative frequencies of the sexes at each stage of development were considered together for the analysis of the reproductive behavior of the population, and, separately, to assess the sexual cycle synchrony between males and females. We observed a variation of sizes between 9.1 and $66.6 \mathrm{~mm}$, with a mean length of $50.2 \mathrm{~mm}$. The study showed no significant difference between the sizes of males and females. There was no evidence of gender differentiation in $2.1 \%$ of subjects analyzed. $51.6 \%$ of subjects were identified as males (M) and $46.3 \%$ as females (F), without significant differences among average number of male and female, resulting in the proportion of M:F ratio of 1,1:1. Lpm was estimated at 11.4 $\mathrm{mm}$, but only to achieve average length of $34.4 \mathrm{~mm}$, all subjects were considered adults. We characterized four stages of evolution of gonadal development in females and males. Analysis of different stages allowed the observation of the atresia phenomena and sex reversal in females. The reproductive cycle presents continuous elimination of gametes, with higher reproductive intensities in the months of November 2001 to April 2002 and also in October 2002.
\end{abstract}

KEYWORDS. Gonadal development, sex ratio, early maturation, atresia, hermaphroditism.

RESUMO. Este estudo teve como objetivos descrever o ciclo gametogênico e o comportamento reprodutivo da população de Iphigenia brasiliana (Lamarck,1818) no estuário do rio Subaé, Baía de Todos os Santos, Bahia. Os bivalves foram coletados de novembro de 2001 a novembro de 2002. Um total de 244 espécimes foi medido (eixo anteroposterior), eviscerado, fixado, desidratado e incluído em parafina. O estudo histológico das gônadas foi realizado através de cortes seriados do tecido gonadal, de $5 \mathrm{~mm}$ de espessura, e corados pela HE. O tamanho médio mínimo da primeira maturação sexual (Lpm) foi estimado a partir da distribuição das frequências relativas de jovens e adultos, por classe de comprimento dos indivíduos. As frequências relativas dos sexos em cada estádio de desenvolvimento foram consideradas conjuntamente para a análise do comportamento reprodutivo da população, e, em separado, para avaliar a sincronia do ciclo sexual entre machos e fêmeas. Foi observada uma variação de tamanhos entre 9,1 e $66,6 \mathrm{~mm}$, com comprimento médio de $50,2 \mathrm{~mm}$. O estudo não demonstrou diferença significativa entre os tamanhos de machos e fêmeas. Não foi possível observar a diferenciação de sexos em 2,1\% dos indivíduos analisados. 51,6\% dos indivíduos foram identificados como machos (M) e 46,3\% como fêmeas (F), não sendo constatadas diferenças significativas entre o número médio de machos e fêmeas, resultando numa proporção de M:F de 1,1:1. O Lpm foi estimado em 11,4 mm, mas apenas ao alcançarem comprimento médio de 34,4 $\mathrm{mm}$, todos os indivíduos foram considerados adultos. Foram caracterizados quatro estádios de evolução do desenvolvimento gonadal em fêmeas e machos. A análise dos diferentes estádios permitiu a observação dos fenômenos de atresia e inversão sexual em fêmeas. O ciclo reprodutivo apresentou eliminação contínua de gametas, com maiores intensidades reprodutivas nos meses de novembro de 2001 a abril de 2002 e, também, no mês de outubro de 2002 .

PALAVRAS-CHAVE. Desenvolvimento gonadal, proporção sexual, primeira maturação, atresia, hermafroditismo.

O conhecimento da biologia reprodutiva de uma espécie é fundamental para compreender a sua história de vida (GARNER et al., 1999) e, também, um requerimento básico para a gestão dos bancos naturais e aplicação de normas de conservação e/ou exploração de espécies marinhas comerciais (Avendaño \& Le PenneC, 1997). Por isso, os ciclos reprodutivos de várias espécies de bivalves têm sido amplamente estudados (NARCHI, 1976; Nascimento \& Luneta, 1978; CAmpos et al., 1998; Gaspar et al., 1999; Pouvreau et al., 2000; Borzone et al., 2001, 2003; RodRíGUEz-RÚa et al., 2003a,b; GIL \& Thomé, 2004a; Morsan \& Kroeck, 2005; SuÁrez et al., 2005; Luz \& BoeHs, 2011).

Iphigenia brasiliana (Lamarck, 1818) é um bivalve conhecido vulgarmente como "tarioba" ou "taioba".
Pertencente à família Donacidae, ocorre em quase toda a costa brasileira, desde o Pará até Santa Catarina, em praias arenosas ou areno-lodosas de baías com influência de água doce ou em estuários (DomAneschi \& Lopes, 1988-89).

Iphigenia brasiliana é um bivalve comestível intensamente explorado por populações humanas que vivem no entorno de regiões estuarinas. Entretanto, apesar da importância econômica, poucos autores estudaram a espécie. NARCHI (1972) descreveu parâmetros morfofuncionais, MEsquita et al. (2001) analisaram a gametogênese em uma população da Lagoa de Itaipu (Niterói, Rio de Janeiro) e CeUTA et al. (2010) evidenciaram hermafroditismo na população da região estuarina do Rio Cachoeira (Ilhéus, Bahia). 
A proporção sexual pode variar entre populações de bivalves devido a fatores como idade do organismo e diferenças genéticas (Morton, 1991); além disso, o ciclo gametogênico pode sofrer influência de parâmetros ambientais (GrotTA \& LunETTA, 1982). Desta forma, não se poderia, simplesmente, extrapolar para Iphigenia brasiliana as características até então descritas para outras espécies, ou mesmo populações desta espécie. Este estudo teve como objetivos descrever o ciclo gametogênico e o comportamento reprodutivo da população de I. brasiliana no estuário do rio Subaé, Baía de Todos os Santos, Bahia.

\section{MATERIAL E MÉTODOS}

As amostragens de Iphigenia brasiliana foram realizadas no estuário do rio Subaé $\left(38^{\circ} 41^{\prime} 21,8^{\prime \prime} \mathrm{W}\right.$ e $12^{\circ} 37^{\prime} 53^{\prime \prime}$ ), contornado por densos manguezais e localizado em São Francisco do Conde, município inserido na Baía de Todos os Santos (BTS), uma das maiores baías do Brasil, com área de $1.086 \mathrm{~km}^{2}$ (LessA et al., 2001).

Os bivalves foram coletados mensalmente, de novembro de 2001 a novembro de 2002 , com exceção dos meses de fevereiro e maio de 2002, tateando-se o substrato semi-submerso. Em laboratório, o comprimento da concha dos indivíduos (eixo anteroposterior) foi medido com paquímetro de precisão de $0,1 \mathrm{~mm}$ e, em seguida, os indivíduos foram mantidos em aquário com aeração constante, entre 24 e 48 horas, com o objetivo de depurar o conteúdo intestinal e da cavidade do manto.

Um total de 244 espécimes foram eviscerados e fixados em líquido de Davidson (Behmer et al., 1976) por 24 horas, desidratados e incluídos em parafina. O estudo histológico da reprodução foi realizado através de cortes seriados $(5 \mathrm{~mm})$ do tecido gonadal e corados pela hematoxilina de Harris e eosina (HE).

Através das preparações histológicas das gônadas, foram identificados e contados os indivíduos de cada sexo e, após confirmação da normalidade da distribuição dos dados pelos testes de Kolmogorov-Smirnoff, os comprimentos de machos e fêmeas foram analisados pelo teste $t$ de Student.

Para a análise da variação temporal da razão sexual, foram estimadas as frequências relativas de machos e fêmeas, verificando a ocorrência de diferença significativa entre as razões sexuais ao longo do tempo, através do teste do qui-quadrado $\left(\chi^{2}\right)$. Os testes estatísticos foram realizados através do programa GraphPad InStat, e os resultados considerados estatisticamente significativos $\operatorname{com} \alpha=0,05$.

O tamanho médio mínimo da primeira maturação sexual (Lpm) foi estimado a partir da distribuição das frequências relativas de jovens (imaturos) e adultos (presença de gametas) por classe de comprimento dos indivíduos, em ambos os sexos, analisados conjuntamente. O Lpm corresponde ao tamanho médio mínimo em que $50 \%$ dos indivíduos iniciam sua participação ativa no ciclo reprodutivo da população (SAntos, 1978).

Para a descrição dos estádios de desenvolvimento gonadal, foi considerada a dominância de um determinado aspecto histológico, de acordo com Nascimento \& Luneta (1978), Tirado \& Salas (1999), RodríGuez-RÚa et al. (2003b) e Gil \& Thomé (2004a). A caracterização dos estádios de desenvolvimento microscópico das gônadas foi baseada na observação (1) da quantidade de tecido conjuntivo interfolicular (espessura das paredes); (2) do grau de desenvolvimento das células gametogênicas; (3) da predominância do tipo citológico gamético no corte; (4) do aspecto de repleção dos folículos ou ácinos gonádicos; (5) da presença de gametas nos gonodutos; e (6) da presença de amebócitos. Quando ocorreu a transição entre um estádio e outro, a classificação foi efetuada considerando-se a dominância do aspecto da citologia dos gametas e da histologia da gônada.

As frequências dos sexos em cada estádio de desenvolvimento gonadal foram consideradas conjuntamente para a análise do comportamento reprodutivo da população e, em separado, para avaliar a sincronia do ciclo sexual entre machos e fêmeas.

\section{RESULTADOS}

Foi observada em Iphigenia brasiliana uma variação de tamanho entre 9,1 e $66,6 \mathrm{~mm}$, com comprimento médio de 50,2 $\mathrm{mm}$. A espécie não apresenta dimorfismo sexual externo, e não houve diferença significativa entre os tamanhos de machos e fêmeas $(\mathrm{t}=0,6024 ; \mathrm{gl}=104 ; \mathrm{p}=0,5482)$. Não foi possível observar a diferenciação de sexos em 5 indivíduos analisados $(2,1 \%)$, nos quais a gônada se encontrava preenchida por tecido conjuntivo, com ausência total de folículos funcionais. Os indivíduos nesse estádio foram considerados indeterminados e observados nos meses de junho, agosto e setembro (Fig. 1).

Dos 244 indivíduos analisados, $126(51,6 \%)$ foram identificados como machos (M) e $113(46,3 \%)$ como fêmeas $(\mathrm{F})$, não sendo constatadas diferenças significativas entre o número médio de machos e fêmeas $\left(\chi^{2}=1,058 ;\right.$ g.l. $\left.=1 ; p>0,05\right)$. Em relação à razão sexual, foi verificada uma proporção de M:F de 1,1:1.

Estimou-se, para a população de Iphigenia brasiliana, o $\mathrm{Lpm}$ quando $50 \%$ dos indivíduos alcançaram cerca de $11,4 \mathrm{~mm}$ de comprimento da concha (Fig. 4), representados na menor classe de tamanho amostrado (9,1-13,7 mm). Foi encontrada uma frequência máxima de adultos com $16,0 \mathrm{~mm}$, embora considerada não significativa por estar representada em um único indivíduo nessa classe de comprimento. Deste modo, apenas ao alcançarem comprimento médio de $34,4 \mathrm{~mm}$, todos os indivíduos foram considerados adultos. 
Foi observado que o conteúdo celular dos folículos ou ácinos gonádicos varia com o desenvolvimento das gônadas, ocorrendo estádios de transição que dão heterogeneidade ao seu aspecto. $\mathrm{O}$ estudo histológico demonstrou células gametogênicas envoltas por um tecido conjuntivo frouxo, do tipo sincicial, que forma a parede dos folículos ou ácinos gonádicos, cuja espessura depende do grau de desenvolvimento gonadal. À proporção que os folículos ou ácinos gonádicos se desenvolvem, o tecido conjuntivo se adelgaça, até tornar-se fino no momento do maior desenvolvimento daquelas estruturas.
Baseado nos aspectos morfológicos observados, foram caracterizados quatro estádios de evolução do desenvolvimento gonadal em fêmeas e machos (Tab. I). Nas fêmeas (Figs 5-8), as ovogônias apresentam grande parte de seu contorno envolto pela parede folicular, enquanto os ovócitos em pré-vitelogênese e em vitelogênese, à medida que se avolumam, vão reduzindo sua zona de contato com a referida parede, projetando-se na luz dos folículos (Fig. 9). A configuração dos ovócitos maduros varia de oval a ligeiramente arredondada. Essas células encontram-se completamente livres na luz dos folículos, enquanto os ovócitos em vitelogênese
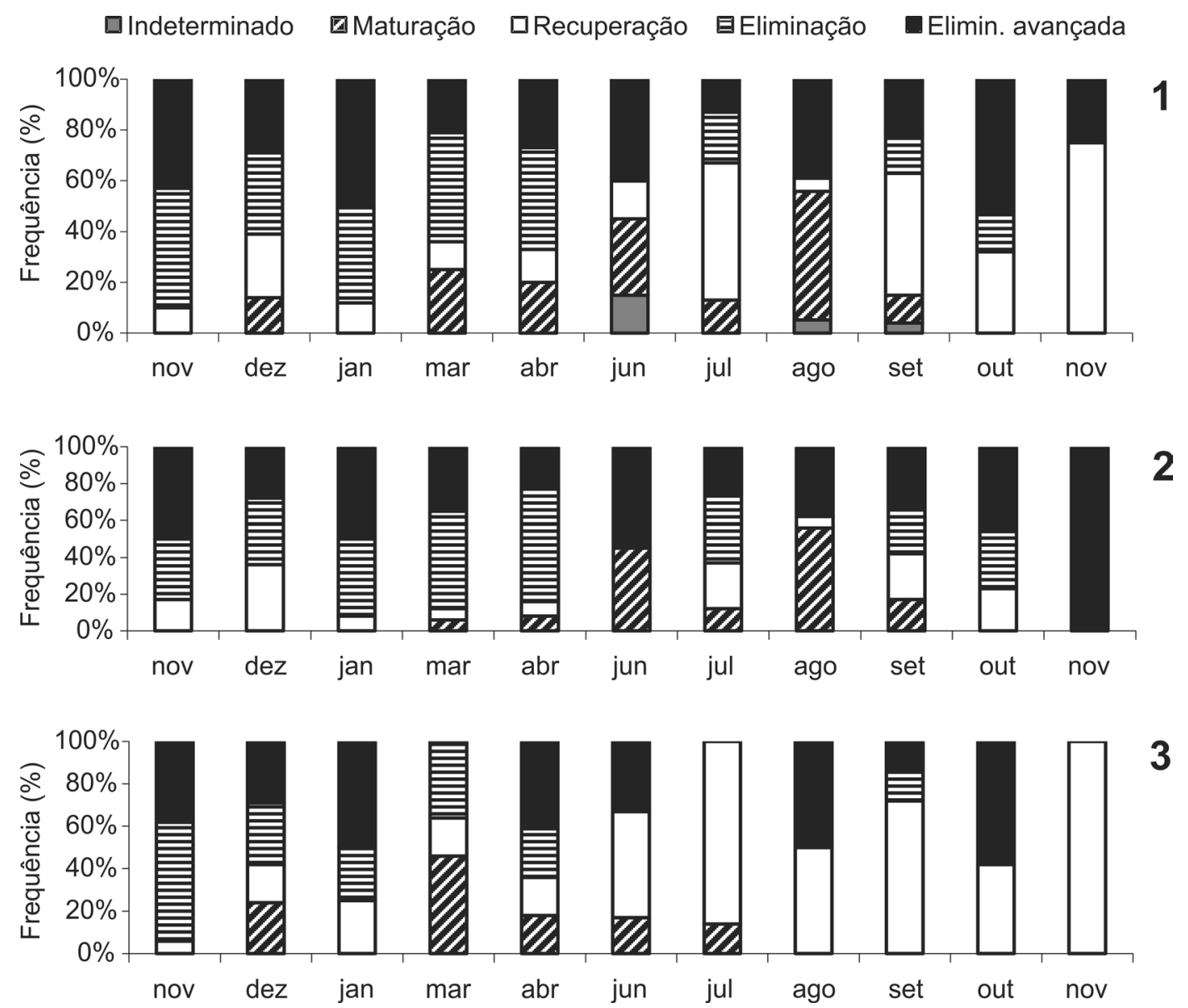

Figs 1-3. Proporção dos estádios de desenvolvimento gonadal de Iphigenia brasiliana (Lamarck, 1818) no estuário do rio Subaé, Bahia: 1, população total; 2 , machos; 3 , fêmeas.

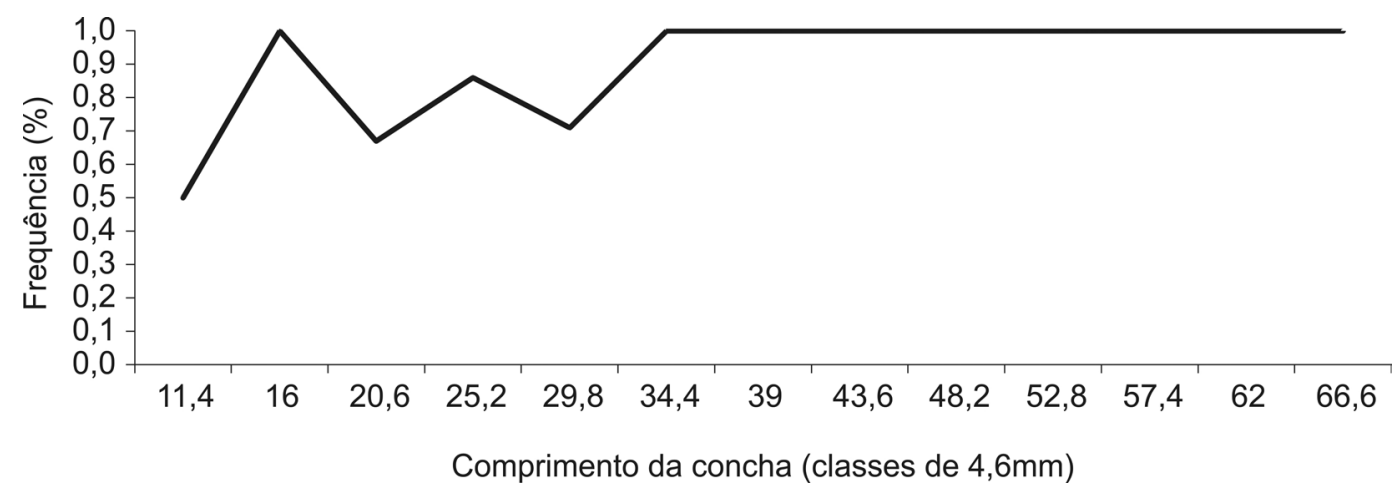

Fig. 4. Tamanho médio mínimo da primeira maturação sexual (Lpm) de Iphigenia brasiliana (Lamarck, 1818) no estuário do rio Subaé, Bahia. 
Tabela I. Estádios de desenvolvimento gonadal feminino e masculino de Iphigenia brasiliana (Lamarck, 1818) no estuário do rio Subaé, Baía de Todos os Santos, Bahia.

\begin{tabular}{|c|c|c|}
\hline \multirow[t]{2}{*}{ Estádios } & \multicolumn{2}{|c|}{ Caracterização dos estádios } \\
\hline & Fêmeas & Machos \\
\hline Maturação & $\begin{array}{l}\text { - Folículos com diâmetro reduzido devido ao pequeno } \\
\text { volume dos gametócitos; } \\
\text { - paredes de tecido conjuntivo espessas e gônias primárias } \\
\text { imersas, em início de desenvolvimento; } \\
\text { - muitos ovócitos em pré-vitelogênese e raros ovócitos } \\
\text { maduros; } \\
\text { - gonodutos vazios, quando visualizados; } \\
\text { - alguns amebócitos interfoliculares. }\end{array}$ & $\begin{array}{l}\text { - Ácinos gonádicos reduzidos; } \\
\text { - grande espaço entre os ácinos em formação; } \\
\text { - bordo do ácino formado pelas células da linhagem } \\
\text { gametogênica inicial; } \\
\text { - raros ou poucos espermatozóides na luz do ácino; } \\
\text { - gonodutos vazios, quando visualizados; } \\
\text { - alguns amebócitos interfoliculares. }\end{array}$ \\
\hline $\begin{array}{l}\text { II - A } \\
\text { Eliminação }\end{array}$ & $\begin{array}{l}\text { - Folículos amplamente distendidos e preenchidos por } \\
\text { ovócitos maduros; } \\
\text { - paredes foliculares adelgaçadas; } \\
\text { - ovócitos maduros presos por um longo pedúnculo, com o } \\
\text { aspecto de uma raquete de tênis, ou livres na luz do folículo; } \\
\text { - gonodutos com ovócitos em eliminação; } \\
\text { - amebócitos não notados. }\end{array}$ & $\begin{array}{l}\text { - Ácinos gonádicos distendidos e volumosos; } \\
\text { - pouco ou nenhum espaço entre os ácinos; } \\
\text { - bordo do ácino com poucas células da linhagem } \\
\text { gametogênica em estádio inicial de desenvolvimento; } \\
\text { - domínio de espermatozóides na luz do ácino; } \\
\text { - gonodutos em eliminação de gametas; } \\
\text { - ocorrência de raros amebócitos. }\end{array}$ \\
\hline $\begin{array}{l}\text { II - B } \\
\text { Eliminação } \\
\text { avançada }\end{array}$ & $\begin{array}{l}\text { - Folículos distendidos com espaços vazios deixados pela } \\
\text { eliminação de gametas; } \\
\text { - paredes do folículo adelgaçadas; } \\
\text { - redução do número de ovócitos maduros no interior dos } \\
\text { folículos; } \\
\text { - início do aparecimento de gônias primárias em } \\
\text { desenvolvimento; } \\
\text { - gonodutos dilatados com poucos gametas em eliminação; } \\
\text { - alguns gametas atrésicos nos folículos e gonodutos; } \\
\text { - aparecimento de alguns amebócitos. }\end{array}$ & $\begin{array}{l}\text { - Aumento dos espaços inter-ácinos pelo esvaziamento dos } \\
\text { gametas; } \\
\text { - início de espessamento da faixa periférica do ácino pelas } \\
\text { células iniciais da linhagem gametogênica; } \\
\text { - aparecimento de vazios intra-ácinos pela eliminação dos } \\
\text { gametas; } \\
\text { - gonoductos contendo poucos espermatozoides em } \\
\text { eliminação; } \\
\text { - presença de amebócitos inter-ácinos. }\end{array}$ \\
\hline $\begin{array}{l}\text { III } \\
\text { Recuperação }\end{array}$ & $\begin{array}{l}\text { - Diminuição do diâmetro dos folículos; } \\
\text { - folículos com paredes bastante espessas; } \\
\text { - presença de gametas maduros residuais e parede folicular } \\
\text { com muitas células em pré-vitelogênese; } \\
\text { - ocorrência de poucos ovócitos atrésicos; } \\
\text { - ocorrência de gonodutos com raros gametas em eliminação; } \\
\text { - presença de amebócitos no interior e ao redor dos folículos; } \\
\text { - ocorrência de inversão sexual. }\end{array}$ & $\begin{array}{l}\text { - Aumento do espaço entre os ácinos pela redução do seu } \\
\text { volume; } \\
\text { - grande quantidade de células iniciais da linhagem } \\
\text { gametogênica; } \\
\text { - presença de espermatozóides residuais; } \\
\text { - gonoductos com raros espermatozóides em eliminação; } \\
\text { - infiltração hemocítica inter-ácino. }\end{array}$ \\
\hline
\end{tabular}

aparecem presos à parede folicular por meio do pedúnculo.

Foi observado que os ovócitos maduros não eliminados entraram em atresia (Fig. 10), ocorrendo, provavelmente, a reabsorção dos gametas. Durante o evento, uma grande massa de material citoplasmático se dispersa com o núcleo e nucléolo, fazendo com que percam a basofilia e tornem-se translúcidos, se comparados aos núcleos normais. A atresia ocorreu nos meses de dezembro de 2001, abril, junho e, com maior frequência, em outubro, de 2012.

Nas gônadas masculinas, as espermatogônias, os espermatócitos e as espermátides aparecem dispostas gradualmente para o interior, no bordo dos ácinos gonádicos, enquanto os espermatozóides são encontrados no centro, com os flagelos voltados para a luz dos folículos (Tab. I; Figs 11-14). A quantidade de citoplasma diminui à medida que as células germinativas vão se desenvolvendo, e a frequência com que aparecem depende do estádio do ciclo sexual em que as gônadas se encontram. Essas células também podem ser diferenciadas pelo aspecto do núcleo. Enquanto as espermatogônias apresentam a cromatina dispersa, nos espermatócitos primários ela aparece na periferia do núcleo, sob a forma de anel, e as espermátides apresentam a cromatina como uma massa densa (Fig. 15).

Em alguns estádios do desenvolvimento gonadal de machos e fêmeas (Tab. I), surgem ao redor dos folículos ou dos ácinos gonádicos numerosos amebócitos, os quais invadem essas estruturas ao término do processo de eliminação.

A análise histológica demonstrou que o ciclo reprodutivo de Iphigenia brasiliana, no estuário do rio Subaé, apresentou variação temporal (Fig. 1), com a ocorrência de eliminação contínua de gametas na população, definindo maiores intensidades reprodutivas nos meses de novembro de 2001 a abril de 2002 e, também, no mês de outubro de 2002. Nesses meses, as frequências obtidas para os estádios de recuperação e maturação apresentaram-se baixas, e de julho a setembro, com um novo pico em novembro de 2002, as gônadas apresentaram maior frequência desses estádios. Assim, as frequências de indivíduos em eliminação e recuperação (levando-se em consideração, também, o estádio de maturação) apresentaram-se numa relação quase inversa.

A análise da distribuição das frequências dos estádios de desenvolvimento gonadal de machos e fêmeas demonstrou um sincronismo quase perfeito entre 


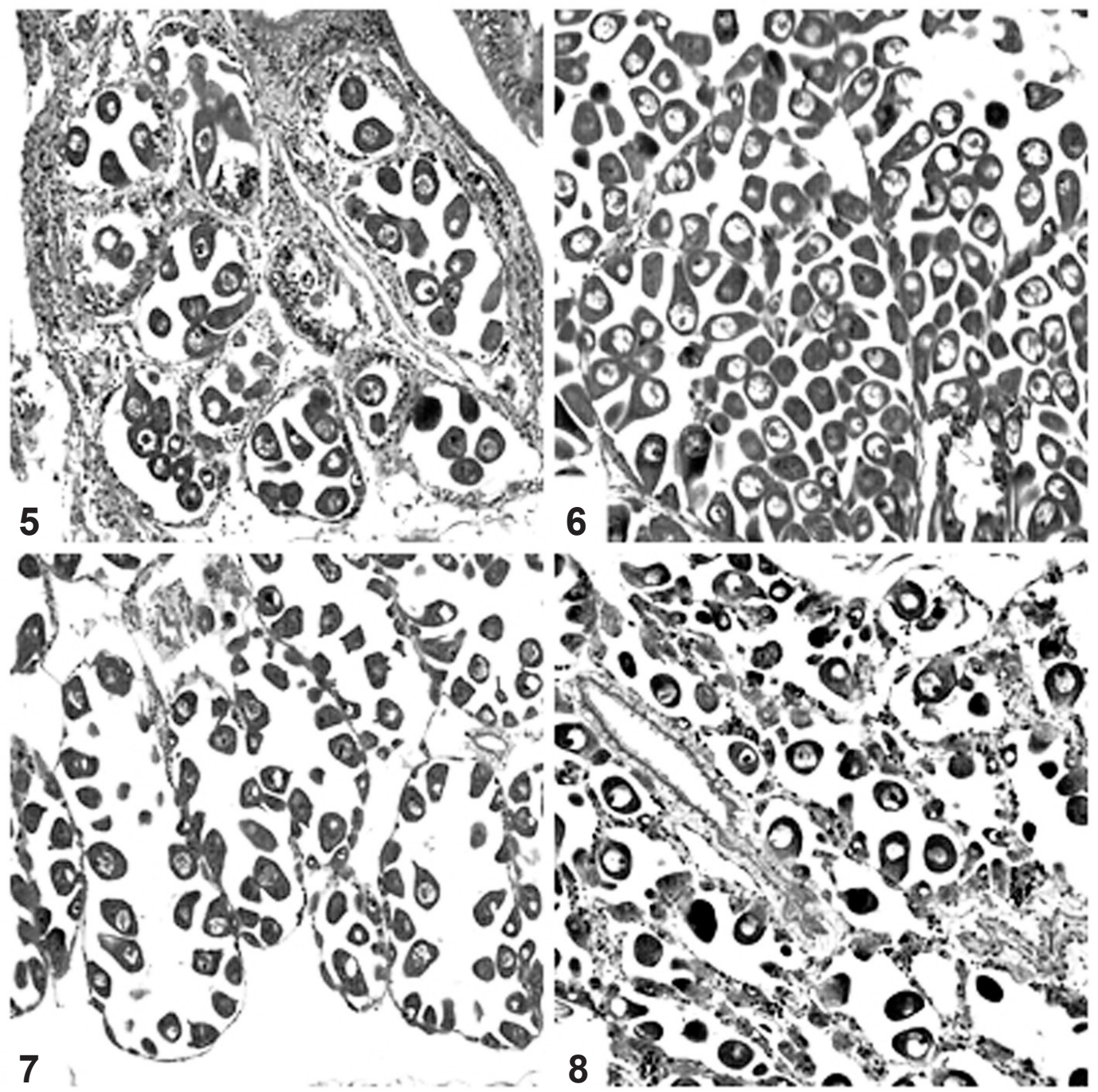

Figs 5-8. Estádios do desenvolvimento gonadal feminino de Iphigenia brasiliana (Lamarck, 1818) no estuário do rio Subaé, Bahia: 5, maturação; 6, eliminação; 7, eliminação avançada; 8, recuperação. 100x.

os sexos, com exceção dos meses de julho e novembro de 2002, nos quais os machos apresentaram estádios de eliminação (Fig. 2), enquanto as fêmeas estavam em maturação e recuperação (Fig. 3). Nos demais meses, foi possível observar que as fêmeas apresentaram uma maior frequência dos estádios de eliminação, enquanto os machos tiveram maiores frequências dos estádios de maturação e recuperação.

Algumas fêmeas apresentaram, no estádio de recuperação, um processo de inversão sexual observado pelas células iniciais da gametogênese masculina (Figs 16-17), sendo constatada a presença de 6,2\% de hermafroditas. Esse processo ocorreu nos meses de novembro de 2001, abril e julho a novembro de 2002.

\section{DISCUSSÃO}

A variação de tamanhos observada nesse estudo contrasta com os dados obtidos por Mesquita et al. (2001) e Ceuta et al.(2010) em estudos na Lagoa de Itaipu (Niterói, Rio de Janeiro) e no estuário do Rio
Cachoeira (Ilhéus, Bahia), respectivamente, pois os menores tamanhos de Iphigenia brasiliana medidos pelos autores estavam entre 41 e 45,7 $\mathrm{mm}$. Provavelmente, esse resultado está relacionado à dificuldade em capturar indivíduos jovens, porque eles cavam rápido e profundamente, de 15 a $20 \mathrm{~cm}$, e submergem em 2 a 3 segundos (Narchi, 1972). Vilanova \& Fonteles-Filho (1989), entretanto, associaram a grande quantidade de indivíduos jovens de Crassostrea rhizophorae (Guilding, 1828 ) ao aumento da salinidade no estuário do rio Ceará (Ceará), o que levou à redução da taxa de crescimento de recrutas. Os autores também associaram essa grande quantidade de indivíduos jovens à estabilização do fluxo da água, a qual facilitou a fixação de recrutas. ARCAs et al. (2001) corroboraram esse resultado ao observarem que a escassa quantidade de recrutas e juvenis de Arca zebra (Swainson, 1883) no Golfo de Cariaco (Venezuela) estava associada à fixação de recrutas em outras áreas, devido às fortes correntes presentes no local.

Outro fator importante para a colonização por novas coortes é a disponibilidade de alimento. TIRADO \& 


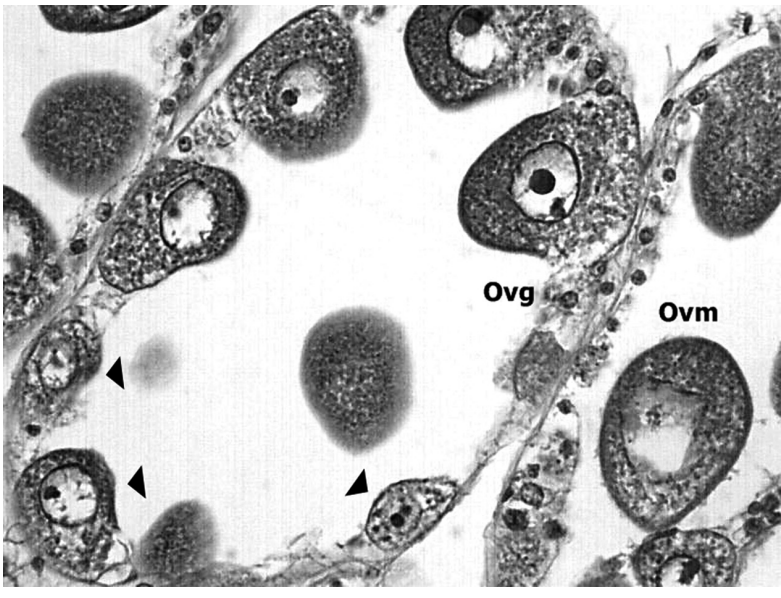

Fig. 9. Células da linhagem gametogênica feminina de Iphigenia brasiliana (Lamarck, 1818) no estuário do rio Subaé, Bahia (Ovg, ovogônia; Ovm, ovócito maduro; cabeça de seta, ovócitos em pré-vitelogênese). 400x.

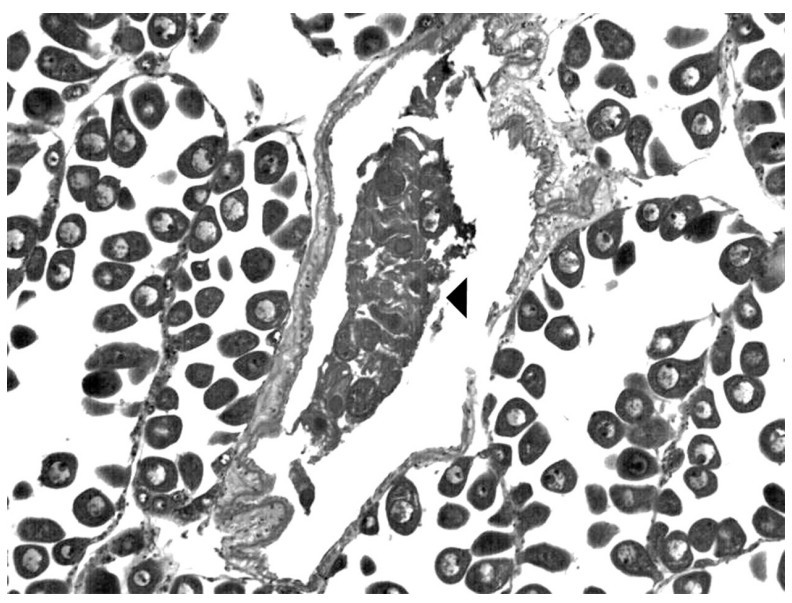

Fig. 10. Gônada feminina de Iphigenia brasiliana (Lamarck, 1818) no estuário do rio Subaé, Bahia, no estádio de eliminação avançada (cabeça de seta, ovócitos atrésicos). 100x.

SAlas (1999), por exemplo, observaram que o aumento de juvenis na população de Donax venustus Poli, 1795 do sul da Espanha coincidia com uma queda significativa na estimativa de matéria orgânica no sedimento, possivelmente devido ao consumo de matéria orgânica por esses juvenis. Para Morsan \& Orensanz (2004), a colonização por novas coortes de Amiantis purpurata (Lamarck, 1818), em estudo realizado na Patagônia (Argentina), provavelmente foi inibida pela alta densidade populacional da espécie.

Iphigenia brasiliana não apresenta dimorfismo sexual externo, como também observado em Chione pubera (Bory Saint-Vicent, 1827) (Borzone et al., 2001), Scrobicularia plana (da Costa, 1778) (RodRíGuez-RúA et al., 2003b) e Donax hanleyanus (Philippi, 1845) (GIL \& Thomé, 2004a). De fato, segundo SAstry (1979), dimorfismo sexual em bivalves é muito raro. Os comprimentos de concha de machos e fêmeas não foram estatisticamente diferentes, corroborando os resultados de BARREIRA \& ARAúJo (2005) para Anomalocardia brasiliana (Gmelin, 1791). Entretanto, esse não é um padrão para bivalves, pois Derbali et al. (2009), em estudo realizado com Cerastoderma glaucum (Poiret, 1789) no Golfo de Gabes (Tunísia), observaram que as fêmeas eram maiores que os machos. Segundo os autores, quando as fêmeas estão em maior número numa população, alocam maior gasto energético para o crescimento, ao invés da reprodução.

Os animais não diferenciados sexualmente apresentaram os comprimentos de concha representados na menor classe de tamanho amostrado, indicando a associação deste estádio com a idade e, portanto, à imaturidade sexual. Outros autores, todavia, relacionaram a presença de indivíduos de sexo indeterminado ao estádio de repouso e início da gametogênese (NASCIMENTO \& Lunetta, 1978; Derbali et al., 2009), ou às grandes variações de salinidade no ambiente e sua influência sobre o metabolismo e diferenciação sexual (GROTTA \& LUNETTA, 1982).

Em animais dioicos, a proporção sexual mais favorável é de 1:1. Diversos estudos com bivalves reforçam a ideia de equilíbrio entre machos e fêmeas (TIRAdO \& SAlas, 1999; Borzone et al., 2001; MorriconI et al., 2002; TORO et al., 2002; RodRÍGUEZ-RÚA et al., 2003a,b; Gil \& Thomé, 2004b; Morsan \& Kroeck, 2005), embora diferenças significativas na proporção sexual também tenham sido encontradas por diferentes autores (Pouvreau et al., 2000; PeÑa et al., 2001; Toro et al., 2002; Mzighani, 2005; SuÁREz et al., 2005). No presente estudo, não foi observada diferença significativa entre o número de machos e fêmeas. Entretanto, foi verificada uma pequena predominância de machos na proporção sexual, em oposição aos resultados obtidos por MESQUITA et al. (2001) e CEUTA et al. (2010) para a espécie, nos quais as fêmeas apareceram em maior número. Segundo Pouvreau et al. (2000), a disponibilidade de alimento favorece o aparecimento de mais fêmeas, caso contrário, seu crescimento é retardado, o que poderia explicar os resultados obtidos nesse estudo. Entretanto, segundo Mzighani (2005), as fêmeas têm maior habilidade para sobreviver em condições ambientais adversas e explica que o maior número de fêmeas observado na população de Anadara antiquata (Linnaeus, 1758) da Tanzânia ocorreu devido ao processo de inversão sexual, no qual os machos estavam se transformando em fêmeas. Levando em consideração o hermafroditismo observado na população de Iphigenia brasiliana desse estudo, em que foi verificado o início da gametogênese masculina em $6,2 \%$ de fêmeas em estádio de recuperação, é possível que o processo de inversão sexual também tenha sido a causa para o maior número de machos observado.

O desenvolvimento das gônadas dos bivalves e o crescimento dos gametas envolvem uma intensa atividade metabólica controlada por fatores externos tais como a temperatura, a salinidade e o alimento (SASTRY, 1979; LunetTa \& Grotta, 1982; Avendaño \& Le PenNeC, 1997; Tirado \& Salas, 1999; Pouvreau et al., 2000; Borzone et al., 2003; RodRíGUEZ-RÚA et al., 2003b; 

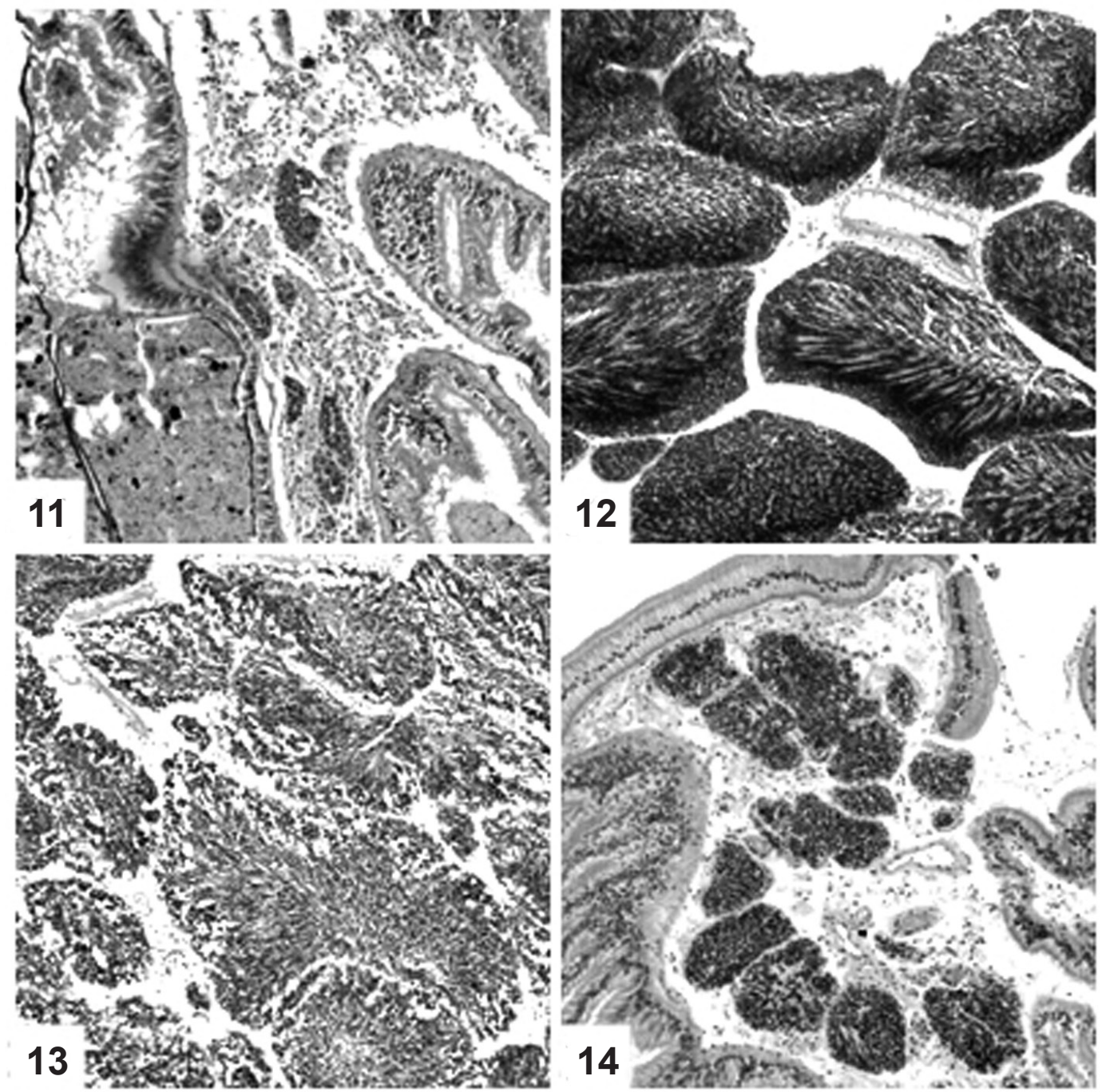

Figs 11-14. Estádios do desenvolvimento gonadal masculino de Iphigenia brasiliana (Lamarck, 1818) no estuário do rio Subaé, Bahia: 11, maturação; 12, eliminação; 13, eliminação avançada; 14, recuperação. 100x.

Gil \& Thomé, 2004a; La Parra et al., 2005; Marenzi \& Branco, 2005; Morsan \& Kroeck, 2005; Suárez et al., 2005), ou por um controle genético da reprodução (Borzone et al., 2003), influindo nos mecanismos de transferência de reservas armazenadas em diversos órgãos para as gônadas.

O tamanho mínimo da primeira maturação sexual em Iphigenia brasiliana $(11,4 \mathrm{~mm})$ foi menor do que o verificado por GIL \& Thомé (2004b) para Donax hanleyanus no litoral norte do Rio Grande do Sul, no qual concluíram que o desenvolvimento da gônada inicia-se num comprimento médio de $11,6 \mathrm{~mm}$ e a maturação sexual, na maioria dos indivíduos, entre 12,0 e 12,8 mm. Entretanto, segundo os autores, a partir desse tamanho, $100 \%$ dos indivíduos apresentam gônadas diferenciadas, enquanto na população de I. brasiliana aqui estudada, somente ao atingir o comprimento de $34,4 \mathrm{~mm}, 100 \%$ dos indivíduos foram considerados maduros. Segundo GIL \& THOMÉ (2004a), a baixa temperatura da água $\left(15^{\circ} \mathrm{C}\right.$ a $18^{\circ} \mathrm{C}$ ) parece exercer influência no processo inicial de maturação. Estudos realizados em anos e pontos diferentes da BTS (Cirano \& Lessa, 2007; OliveiraSilva et al., 2008; Milazzo et al., 2011), demonstraram que a temperatura da água varia entre $25^{\circ}$ e $32,7^{\circ} \mathrm{C}$, sendo considerada uma variável de médias elevadas e de

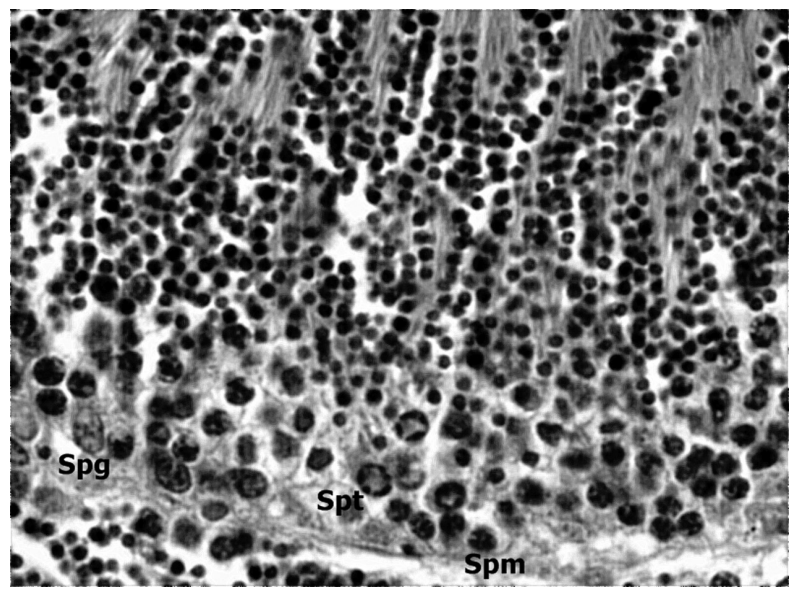

Fig. 15. Células da linhagem gametogênica masculina de Iphigenia brasiliana (Lamarck, 1818) no estuário do rio Subaé, Bahia (Spg, espermatogônia; Spm, espermátide; Spt, espermatócito primário). 1000x. 


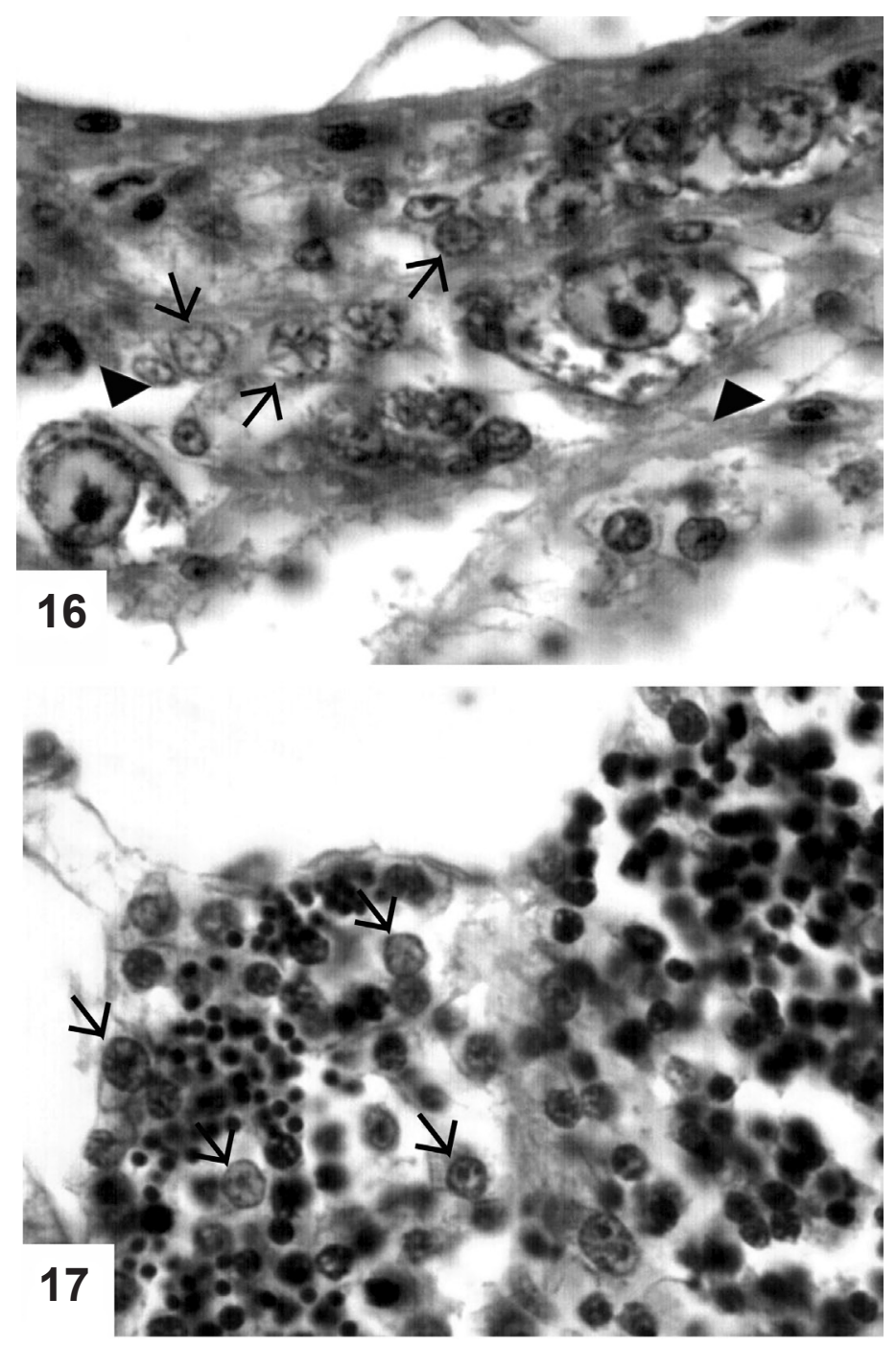

Fig. 16-17. Hermafroditismo em Iphigenia brasiliana (Lamarck, 1818) no estuário do rio Subaé, Bahia: 16, gônada feminina no estádio de recuperação, com células iniciais da gametogênese masculina; 17, gônada masculina com células em diferentes estádios da gametogênese (cabeça de seta, ovócitos em pré-vitelogênese; seta, células iniciais da linhagem gametogênica masculina). 1000x.

pequena amplitude em toda essa região (PAREDES et al., 1980), o que pode ter provocado um atraso na maturação inicial da população.

$\mathrm{O}$ estudo demonstrou que existe semelhança entre características do desenvolvimento gonadal de I. brasiliana e outros bivalves, sugerindo certa padronização do ciclo gametogênico em Bivalvia. Foi observado que nas gônadas masculinas e femininas de I. brasiliana quase sempre estavam presentes todas as células da linhagem gametogênica, independente do estádio de desenvolvimento gonadal, como também observado por MoRRICONI et al. (2002) para Eurhomalea exalbida (Chemnitz, 1795) e Gil \& Thоме́ (2004a) para Donax hanleyanus. Entretanto, LAVENDER et al. (2011), ao estudarem a população de Anomalocardia brasiliana no litoral norte de Pernambuco, constataram que cada estádio do desenvolvimento gonadal apresentou peculiaridades em ambos os sexos, o que determinou características celulares diferentes entre os estádios.

Nas fêmeas de Iphigenia brasiliana, o estádio de recuperação evidencia folículos com paredes espessas, com muitas células em pré-vitelogênese, e a presença de gametas maduros residuais, como registrado em Anomalocardia brasliliana por NARCHI (1976). Foi observado que as gônadas dos indivíduos, de ambos os sexos, no estádio de maturação, apresentaram características muito parecidas com o estádio de recuperação, fato também observado por LAMMENS (1967).

Assim como verificado neste estudo, outros autores também observaram processos degenerativos de gametas femininos em bivalves (TIRADO \& SAlaS, 1999; Borzone et al., 2003), os quais poderiam ser uma resposta à liberação de espaço intrafolicular para a proliferação de gametas. SASTRY (1979) afirmou que moluscos expostos a baixas salinidades por tempo prolongado apresentam reabsorção gonadal. De acordo com SuÁREZ et al.(2005), em condições desfavoráveis à eliminação dos ovócitos maduros, esses permanecem no lúmen do folículo e são reabsorvidos. Entretanto, essa 
evidência não corrobora as observações deste trabalho, pois outubro, mês de maior frequência do processo de atresia, também foi o de maior frequência de fêmeas em estádio de eliminação avançada. Desse modo, como foi verificado que em outubro as fêmeas também estavam em recuperação e, no mês seguinte, ocorreu uma frequência de $100 \%$ de fêmeas nesse estádio, a atresia, possivelmente, esteja relacionada à liberação de espaço intrafolicular para a produção de novos gametas. Durante o processo de atresia, os ovócitos adquirem formato irregular ou amebóide, concentrando-se no centro do folículo ou nos gonodutos (BORZONE et al., 2003). A destruição dos folículos, então, ocorre pelos lisossomos produzidos durante a vitelogênese e pelas enzimas liberadas pela quebra da parede do ovócito (Avendaño \& Le Pennec, 1997).

Em machos de I. brasiliana não foi possível observar espermatócitos secundários. SuÁrez et al. (2005) também tiveram certa dificuldade para observar os espermatócitos secundários em Mytilus galloprovincialis (Lamark 1819), devido à rapidez em que ocorre a segunda divisão meiótica. No entanto, Chatchavalvanich et al. (2006) encontraram espermatócitos secundários em Hyriopsis (Hyriopsis) bialatus Simpson, 1900 após a conclusão da primeira divisão meiótica, descrevendo-os como células menores que os espermatócitos primários, com núcleos mais condensados.

A presença de amebócitos parece estar associada com a reabsorção dos gametas residuais. MORRICONI \& Calvo (1978) encontraram amebócitos em Ostrea puelchana d'Orbigny, 1841 no início da maturação e começo da etapa pós-desova. Além de função reprodutiva, de acordo com NASCiMENTO \& LunETTA (1978), eles desempenham um papel muito importante nos processos da digestão e excreção dos bivalves em geral, uma vez que são capazes de realizar a fagocitose sem a formação de vacúolos.

Não foi presenciado um estádio de repleção total, no qual os ovócitos preenchem toda a área alveolar, corroborando com os estudos de MORRICONI et al. (2002) com Eurhomalea exalbida e MORSAN \& KROECK (2005) com Amiantis purpurata. $\mathrm{O}$ processo de eliminação de gametas inicia-se com o esvaziamento parcial da gônada, como também constatado por MORRICONI et al. (2002) e Borzone et al. (2003) em Euvola ziczac (Linneaus, 1758). Em seguida, o estádio de esvaziamento avançado se instala, e logo após o estádio de recuperação, quando o ciclo retorna com a gônada no estádio de maturação. Vários autores (NARChI, 1976; NASCIMENTo \& LunetTA, 1978; Avendaño \& Le Pennec, 1997; Pouvreau et al., 2000; Borzone et al., 2001; MoRriconi et al., 2002; Borzone et al., 2003; GIL \& Thomé, 2004a; SuÁrez et al., 2005; Luz \& Boens, 2011) também constataram que diferentes espécies passam da condição de eliminação para início de desenvolvimento de novos ovócitos sem que haja um período de repouso definido, como descrito por RODRíGUEZ-RÚA et al. (2003b) para Scrobicularia plana. O ciclo reprodutivo contínuo testemunhado para machos e fêmeas de Iphigenia brasiliana pode estar relacionado às temperaturas mais ou menos elevadas e constantes (Luz \& BoEHs, 2011) da água da BTS, aumentando o sucesso reprodutivo (Toro et al., 2002) através da redução da possibilidade de uma falha no recrutamento da população e sua consequente extinção, principalmente para aquelas espécies de menor longevidade (Borzone et al., 2003). Para Pouvreau et al. (2000), a eliminação contínua de gametas é uma estratégia oportunista que consiste em investir energia extra para a produção de gametas, mesmo que os parâmetros ambientais sejam restritivos à gametogênese. Ele conclui que as espécies com ciclo sexual contínuo apresentam as seguintes características histológicas: (1) folículos, na mesma gônada, em diferentes estádios de maturação; (2) gametogênese como um processo rápido e sempre ativo e (3) a eliminação é, geralmente, incompleta. Essas características também foram observadas para a população de I. brasiliana deste estudo.

As frequências de machos em maturação e recuperação foram menores que as de fêmeas durante o estudo. Nascimento \& LunetTa (1978) sugeriram que, em Crassostrea rhizophorae, os processos de recuperação e maturação são mais rápidos nos machos, por isso são raramente detectados. Mzighani (2005) e SuÁREZ et al. (2005) também encontraram fêmeas proliferando mais lentamente que os machos e sincronia entre os sexos no estádio de eliminação de gametas.

Neste estudo, foi possível observar que 6,2\% dos indivíduos eram hermafroditas, embora Ceuta et al. (2010) tenham constatado hermafroditas em apenas $0,2 \%$ da população dessa espécie no estuário do rio Cachoeira (Ilhéus/Bahia), relacionando-a a possíveis variações de salinidade e poluição, já que, segundo os autores, os fatores que determinam o hermafroditismo em bivalves dioicos ainda não são bem conhecidos.

A literatura evidencia diversos casos de hermafroditismo em bivalves (HELleR, 1993; AvendAÑo \& Le Pennec, 1997; Borzone et al., 2003; Morsan \& Kroeck, 2005; Petersen et al., 2008; Ceuta et al., 2010), podendo ser uma protandria (LüTZEN et al., 2001; Mzighani, 2005; Chesman \& Langston, 2006) ou protoginia (LEONARD, 1969). A protandria confere aos machos uma vantagem na competição para a fertilização de ovócitos altamente férteis (Mzighani, 2005). De acordo com Heller (1993), o hermafroditismo é vantajoso para indivíduos que têm dificuldade em encontrar um parceiro numa determinada população.

Os casos de hermafroditismo em bivalves têm sido associados à mudança de condições ambientais (Pouvreau et al., 2000; Ceuta et al., 2010), mecanismos genéticos (Mzighani, 2005) ou infestação por parasitas (Heller, 1993). Neste estudo, bem como nos estudos de MesQuita et al. (2001) e Ceuta et al. (2010), todavia, não foram encontrados parasitas, possivelmente por uma maior resistência da espécie aos parasitas comumente encontrados em outros bivalves.

Chesman \& Langston (2006) observaram que 
houve feminização de machos de Scrobicularia plana expostos a contaminantes químicos capazes de provocar desregulação endócrina. Diferentes estudos realizados na BTS demonstram contaminação local por óleo e derivados (Celino \& Queiroz, 2006), metais pesados (AMAdo-Filho et al., 2008) e resíduos sólidos (CARVALHOSouZA \& Tinôco, 2011), o que pode ter contribuído para o elevado índice de hermafroditismo encontrado na população estudada.

O estudo histológico das gônadas de Iphigenia brasiliana do estuário do rio Subaé permitiu concluir que a espécie possui um ciclo reprodutivo contínuo, com picos mais intensos de eliminação de gametas nos meses de novembro de 2001 a abril de 2002 e outubro do mesmo ano. Há um sincronismo quase perfeito entre machos e fêmeas no que diz respeito à frequência dos estádios do ciclo sexual. Entretanto, estudos com maior periodicidade na área, associando o ciclo gametogênico às variáveis ambientais, são recomendados para uma avaliação mais precisa do comportamento reprodutivo da espécie.

Agradecimentos. Ao Dr. Antônio Fernando de Souza Queiroz pela viabilização da execução desse trabalho com o apoio da FINEP/ CTPETRO, através do convênio 6400028700.

\section{REFERÊNCIAS BIBLIOGRÁFICAS}

Amado-Filho, G. M.; Salgado, L. T.; Rebelo, M. F.; Rezende, C. E.; Karez, C. S. \& Pfeiffer, W. C. 2008. Heavy metals in benthic organisms from Todos os Santos Bay, Brazil. Brazilian Journal of Biology 68(1):95-100.

Arcas, A. P.; Ramos A. O.; Arrieche, D.; Villalba, J. \& Lodeiros, C. 2001. Producción secundaria y indice de condición en Arca zebra (Mollusca: Bivalvia) del Golfo de Cariaco, Venezuela. Revista de Biología Tropical 49(2):599-608.

Avendaño, M. \& Le Pennec, M.1997. Intraspecific variation in gametogenesis in two populations of the Chilean molluscan bivalve, Argopecten purpuratus (Lamarck). Aquaculture Research 28:175-182.

Barreira, C. A. R. \& ARAúJo, M. L. R. 2005. Ciclo reprodutivo de Anomalocardia brasiliana (Gmelin, 1791) (Mollusca, Bivalvia, Veneridae) na Praia do Canto da Barra, Fortim, Ceará, Brasil. Boletim do Instituto de Pesca 31(1):9-20.

Behmer, O. A.; Tolosa, E. M. C. \& Freitas de Neto, A. G. 1976. Manual de Técnicas para Histologia Normal e Patologia. São Paulo, EDART - Editora da USP. 256p.

Borzone, C. A.; Pezzuto, P. R. \& Tavares, Y. A. G. 2003. Características histológicas del ciclo reproductivo de Euvola ziczac (Linnaeus) (Pectinidae, Bivalvia) del litoral sur-sudeste del Brasil. Revista Brasileira de Zoologia 20(4):763-772.

Borzone, C. A.; Vargas, K. M.; Pezzuto, P. R. \& Tavares, Y. A. G. 2001. Aspectos da reprodução e dinâmica populacional de Chione pubera (Bory Saint-Vicent) (Bivalvia, Veneridae) no sul do Brasil. Revista Brasileira de Zoologia 18(2):333-349.

Campos, E. R.; Peña, J. C.; Cruz, R. A. \& Palacios, J. A. 1998. Crecimiento y ciclo reproductivo de Polymesoda radiata (Bivalvia: Corbiculidae) en Costa Rica. Revista de Biología Tropical 46(3):643-648.

Carvalho-Souza, G. F. \& Tinôco, M. S. 2011. Avaliação do lixo marinho em costões rochosos na Baía de Todos os Santos, Bahia, Brasil. Revista da Gestão Costeira Integrada 11(1):135-143.

Celino, J. J. \& Queiroz, A. F. DE S. 2006. Fonte e grau da contaminação por hidrocarbonetos policíclicos aromáticos (HPAs) de baixa massa molecular em sedimentos da baía de Todos os Santos, Bahia. Revista Escola de Minas 59(3):265-270.

Ceuta, L. O.; Boehs, G. \& Santos, J. J. B. 2010. Hermaphroditism among dioecious Tagelus plebeius (Lightfoot, 1786) (Mollusca,
Psammobiidae) and Iphigenia brasiliana (Lamarck, 1818) (Mollusca, Donacidae) on the Cachoeira River Estuary, Ilhéus, Bahia, Brazil. Brazilian Journal of Biology 70(1):125-127.

Chatchavalvanich, K.; Jindamongkon, P.; Kovitvadhi, U.; Thongpan, A. \& KovitvadHI, S. 2006. Histological structure of gonads in the freshwater pearl mussel, Hyriopsis (Hyriopsis) bialatus Simpson, 1900. Invertebrate Reproduction and Development 49(4):245253.

Chesman, B. S. \& Langston, W. J. 2006. Intersex in the clam Scrobicularia plana: a sign of endocrine disruption in estuaries? Biology Letters 2:420-422.

Cirano, M. \& Lessa, G. C. 2007. Oceanographic characteristics of Baía de Todos os Santos, Brazil. Revista Brasileira de Geofísica 25(4):363-387.

Derbali, A.; Jarboui, O. \& Ghorbel, M. 2009. Reproductive biology of the cockle Cerastoderma glaucum (Mollusca: Bivalvia) from the north coast of Sfax (Gulf of Gabes, Tunisia). Ciencias Marinas 35(2): 141-152.

Domaneschi, O. \& Lopes, S. G. B. C. 1988-89. Família Donacidae Fleming, 1828. Informativo SBM 87:9-12; 88:9-12; 92:9-12; 93 9-12.

Garner, J. T.; Haggerty, T. M. \& Modlin, R. F. 1999. Reproductive cycle of Quadrula metanevra (Bivalvia: Unionidae) in the Pickwick Dam tailwater of the Tennessee River. The American Midland Naturalist 141:277-283.

Gaspar, M. B.; Ferreira, R. \& Monteiro, C. C. 1999. Growth and reproductive cycle of Donax trunculus L. (Mollusca: Bivalvia) off Faro, southern Portugal. Fisheries Research 41:309-316.

GiL, G. M. \& ThомÉ, J. W. 2004a. Descrição do ciclo reprodutivo de Donax hanleyanus (Bivalvia, Donacidae) no sul do Brasil. Iheringia, Série Zoologia 94(3):271-276.

. 2004b. Proporção sexual e comprimento de concha na primeira maturação sexual em Donax hanleyanus Philippi (Bivalvia, Donacidae) no Rio Grande do Sul, Brasil. Revista Brasileira de Zoologia 21(2):345-350.

Grotta, M. \& Lunetta, J. E. 1982. Reproductive physiological variation of Anomalocardia brasiliana (Gmelin, 1791) (Mollusca - Bivalvia), in different latitudes. Revista Nordestina de Biologia 5(1):21-28

Heller, J. 1993. Hermaphroditism in molluscs. Biological Journal of the Linnean Society 48:19-42.

Lammens, J. J. 1967. Growth and Reproduction a Tidal Flat Population of Macoma balthica (Linnaeus, 1758). Netherlands Journal of Sea Research 3:315-382.

La Parra, A. M.; García, O. \& SAn Juan, F. 2005. Seasonal variations on the biochemical composition and lipid classes of the gonadal and storage tissues of Crassostrea gigas (Thunberg, 1794) in relation to the gametogenic cycle. Journal of Shellfish Research 24(2):457-467.

Lavander, H. D.; Cardoso Júnior, L. O.; Oliveira, R. L.; Silva Neto, S. R.; Olivera Galvez, A.\& Peixoto, S. R. M. 2011. Biologia reprodutiva da Anomalocardia brasiliana (Gmelin, 1791) no litoral norte de Pernambuco, Brasil. Revista Brasileira de Ciências Agrárias 6(2):344-350.

Leonard, V. K. J. 1969. Seasonal Gonadal Changes in Two Bivalve Mollusks in Tomales Bay, California. The Veliger 11(4):382-390.

Lessa, G. C.; Dominguez, J. M. L.; Bittenourt, A. C. S. P. \& Brichta, A. 2001. The tides and tidal of Todos os Santos Bay, Northeast Brazil: a general characterization. Anais da Academia Brasileira de Ciências 73(2):245-261.

Lunetta, J. E. \& Grotta, M. 1982. Influência dos fatores exógenos e endógenos sobre a reprodução de moluscos marinhos. Boletim de Fisiologia Animal 6:191-204.

LÜtZen, J.; TAKahashi, T. \& Yamaguchi, T. 2001. Morphology and reproduction of Nipponomysella subtruncata (Yokoyama), a galeommatoidean bivalve commensal with the sipunculan Siphonosoma cumanense (Keferstein) in Japan. Journal of Zoology 254:429-440.

Luz, J. R. \& Boens, G. 2011. Reproductive cycle of Anomalocardia brasiliana (Mollusca: Bivalvia: Veneridae) in the estuary of the Cachoeira River, Ilhéus, Bahia. Brazilian Journal of Biology 71(3):679-686.

Marenzi, A. \& Branco, J. 2005. O mexilhão Perna perna (Linnaeus) (Bivalvia, Mytilidae) em cultivo na Armação do Itapocoroy, Santa Catarina, Brasil. Revista Brasileira de Zoologia 22(2):394-399. 
Mesquita, E. F. M.; Abreu, M. G. \& Lima, F. C. 2001. Aspectos gametogênicos e histoquímicos de Iphigenia brasiliana (Lamarck) (Bivalvia, Donacidae) da Lagoa de Itaipu, Niterói, Rio de Janeiro, Brasil. Revista Brasileira de Zoologia 18(2):523-528.

Milazzo, A. D. D.; Rios, M. C.; Otero, O. M. F. \& Cruz, M. J. M. 2011. Concentração de metais em águas superficiais do estuário do Rio São Paulo, Baía de Todos os Santos. Cadernos de Geociências 8(1):42-46.

Morriconi, E. \& Calvo, J. 1978. Ciclo reproductivo y alternancia de sexos en Ostrea puelchana. Physis, Seccion A 38(95):1-7.

Morriconi, E.; Lomovasky, B. J.; Calvo, J. \& Brey, T. 2002. The reproductive cycle of Eurhomalea exalbida (Chemnitz, 1795) (Bivalvia: Veneridae) in Ushuaia Bay (54 50 'S), Beagle Channel (Argentina). Invertebrate Reproduction and Development 42:61-68.

Morsan, E. M. \& Kroeck, M. A. 2005. Reproductive cycle of purple clam, Amiantis purpurata (Bivalvia: Veneridae) in northern Patagonia (Argentina). Journal of the Marine Biological Association of the United Kingdom 85:367-373.

Morsan, E. M. \& Orensanz, J. M. 2004. Age structure and growth in an unusual population of purple clam, Amiantis purpurata (Lamarck, 1818) (Bivalvia: Veneridae), from Argentine Patagonia. Journal of Shellfish Research 23(1):73-80.

Morton, B. 1991. Do the Bivalvia demonstrate environment-specific sexual strategies? A Hong Kong Model. Journal of Zoology 223:131-142.

Mzighani, S. 2005. Fecundity and population structure of cockles, Anadara antiquate L. 1758 (Bivalvia: Arcidae) from a sandy / muddy beach near Dar es Salaam, Tanzania. Western Indian Ocean Journal of Marine Science 4(1):77-84.

NARCHI, W. 1972. On the Biology of Iphigenia brasiliensis Lamarck, 1818 (Bivalvia, Donacidae). Proceedings Malacological Society of London 40:79-91.

1976. Ciclo anual da gametogênese de Anomalocardia brasiliana (Gmelin, 1791) (Mollusca Bivalvia). Boletim de Zoologia da Universidade de São Paulo 1:331-350.

Nascimento, I. A. \& Lunetta, J. E. 1978. Ciclo sexual da ostra de mangue e sua importância para o cultivo. Boletim de Fisiologia Animal 2:63-98

Oliveira-Silva, J. T.; Peso-Aguiar, M. C. \& Lopes, P. R. D. 2008. Ictiofauna das praias de Cabuçu e Berlinque: uma contribuição ao conhecimento das comunidades de peixes na Baía de Todos os Santos - Bahia - Brasil. Biotemas 21(4):105-115.

Paredes, J. F.; Peixinho, V. M. C. \& Brito, R. R. C. 1980. Produtividade primária, biomassa e fatores limitantes na área estuarina sw da Baía de Todos os Santos. Boletim do Instituto de Oceanografia 29(2):275-282.

Peña, J. H. C.; Quesada, M. P.; Hernández, M. U. \& Vargas, O. S. 2001.Crecimiento y madurez sexual de una población de Saccostrea palmula (Mollusca: Bivalvia), Costa Rica. Revista de Biología Tropical 49(3-4):877-882.

Petersen, J. L.; Ibarra, A. M.; Ramirez, J. L. \& May, B. 2008. An induced mass spawn of the hermaphroditic Lion-Paw Scallop, Nodipecten subnodosus: genetic assignment of maternal and paternal parentage. Journal of Heredity 99(4):337-348.

Pouvreau, S.; Gangnery, A.; Tiapari, J.; Lagarde, F.; Garnier, M. \& Bodoy, A. 2000. Gametogenic cycle and reproductive effort of the tropical blacklip pearl oyster, Pinctada margaritifera (Bivalvia: Pteriidae), cultivated in Takapoto atoll (French Polynesia) Aquatic Living Resources 13(1):37-48

Rodríguez-Rúa, A.; Prado, M. A. \& Bruzón, M. A. 2003a. Estudio del ciclo reproductor de Chamelea gallina (L., 1758) (Mollusca: Bivalvia) en tres poblaciones del litoral andaluz. Boletin del Instituto Español de Oceanografia 19(1-4):57-63.

Rodríguez-Rúa, A.; Prado, M. A.; Romero, Z. \& Bruzón, M. A. 2003b. The gametogenic cycle of Scrobicularia plana (da Costa 1778) (Mollusca: Bivalve) in Guadalquivir estuary (Cádiz, SW Spain). Aquaculture 217:157-166.

SAnTos, E. P. 1978. Dinâmica de populações aplicada à pesca piscicultura. São Paulo, Hucitec, EDUSP. 129p.

Sastry, A. N. 1979. Pelecypoda (excluding ostreidae). In: Giese, A. C. \& Pearse, J. S. eds. Reproduction of Marine Invertebrates. vol. 5. New York, Academic Press. p.113-292.

Suárez, M.; Alvarez, C.; Molist, P. \& San Juan, F. 2005. Particular aspects of gonadal cycle and seasonal distribution of gametogenic stages of Mytilus galloprovincialis cultured in the estuary of Vigo. Journal of Shellfish Research 24(2):531-540.

Tirado, C. \& Salas, C. 1999. Reproduction of Donax venustus Poli 1795, Donax semistriatus Poli 1795 and intermediate morphotypes (Bivalvia: Donacidae) in the littoral of Málaga (Southern Spain). Marine Ecology 20(2):111-130.

Toro, J. E.; Thompson, R. J.; InNes, D. J. 2002. Reproductive isolation and reproductive output in two sympatric mussel species (Mytilus edulis, M. trossulus) and their hybrids from Newfoundland. Marine Biology 141:897-909.

Vilanova, M. F. V. \& Fonteles-Filho, A. A. 1989. Análise da biometria e do fator de condição da ostra-do-mangue, Crassostrea rhizophorae (Guilding, 1828) (Mollusca, Bivalvia), no estuário do rio Ceará, Ceará, Brasil. Ciência e Cultura 41(11):1117-1124. 